\title{
Cardiovascular risk assessment prior to kidney transplantation
}

\author{
Vnucak $\mathrm{M}^{1}$, Granak $\mathrm{K}^{1}$, Skalova $\mathrm{P}^{1}$, Laca L ${ }^{1}$, Mokan $\mathrm{M}^{2}$, Dedinska $\mathrm{I}^{1}$ \\ Department of Surgery and Transplantation Centre, University Hospital Martin and Jessenius \\ Medical Faculty of Comenius University, Martin, Slovakia. granak.k@gmail.com
}

\begin{abstract}
OBJECTIVES: The objective was to find out risk factors indicating the patients directly to selective coronarography (SCG) to avoid unnecessary non-invasive testing and in their absence to asses low cardiovascular risk and faster inclusion on the waiting list.

BACKGROUND: Cardiovascular diseases (CVD) are the most frequent cause of death in dialysed patients. The aim of our retrospective analysis was to identify risk factors for coronary artery disease (CAD) before kidney transplantation (KTx).

METHODS: Our retrospective analysis consisted of 55 dialysed patients (46 males, 9 females, $p<0.0001$ ), undergoing SCG before KTx. We divided the patients according to SCG results (negative, $n=40$, positive finding, $n=15$ ).

RESULTS: We confirmed a significantly lower incidence of diabetic nephropathy $(p=0.0484)$, ischaemic heart disease $(p=0.0174)$ and CAD $(p=0.0001)$ in patients without percutaneous coronary intervention (PCl; negative finding). Haemodynamically significant coronary stenosis correlated with the occurrence of stroke in a patient's history $(p=0.0104)$. We identified predictors for performing $\mathrm{PCl}$ (positive result): type 2 diabetes mellitus (DM) $(p=0.0472)$, high-density lipoprotein cholesterol $\leq 1.03 \mathrm{mmol} / \mathrm{l}(p=0.0359)$, total calcium level $\leq 2 \mathrm{mmol} / \mathrm{l}(p=0.0309)$, phosphate level $\geq 1.45 \mathrm{mmol} / \mathrm{l}(\mathrm{OR} 0.2034 ; p=0.0351)$.

CONCLUSION: In our analysis, patients with DM and poorly managed chronic kidney disease/mineral bone disease were the riskiest subset of the patients with a positive SCG finding (Tab. 4, Fig. 2, Ref. 30). Text in PDF www.elis.sk

KEY WORDS: kidney transplantation, coronary artery disease, selective coronarography, cardiovascular risk.
\end{abstract}

\begin{abstract}
Abbreviations: ACC/AHA - American College of Cardiology/ American Heart Association, ACEi - angiotensin converting enzyme inhibitor, ACS - acute coronary syndrome, AGEP - advanced glycated end products, ARB - angiotensin II receptor blockers, ASA - aminoacyl salicylic acid, BMI - body mass index, CAD - coronary artery disease, $\mathrm{CKD}$ - chronic kidney disease, $\mathrm{CKD}$ / MBD - chronic kidney disease/mineral bone disease, CVD - cardiovascular diseases, DM - diabetes mellitus, DSE - dobutamine stress echocardiography, ECG - electrocardiogram, ESC - European Society of Cardiology, ESRD - end stage renal disease, HD - haemodynamically, HDL - high-density lipoprotein, IVS - interventricular septum, KTC - kidney transplant candidate, KTx - kidney transplantation, LDL - low-density lipoprotein, MET - metabolic equivalent, PCI - percutaneous coronary intervention, $\mathrm{PTH}$ - parathormone, SCG - selective coronarography, TAG - triacylglycerides, TIA - transitory ischaemic attack, TTE - transthoracic echocardiogram, WL - waiting list
\end{abstract}

${ }^{1}$ Department of Surgery and Transplantation Centre, University Hospital Martin and Jessenius Medical Faculty of Comenius University, Martin, Slovakia, and ${ }^{2} 1$ stDepartment of Internal Diseases, University Hospital Martin and Jessenius Medical Faculty of Comenius University, Martin, Slovakia

Address for correspondence: K. Granak, MD, Department of Surgery and Transplantation Center, University Hospital Martin, Kollarova 2, SK-036 01 Martin, Slovakia. Phone: +421.903604190

\section{Introduction}

Patients with chronic kidney disease (CKD) have a very high incidence of acute cardiovascular events that result from the presence of traditional and non-traditional risk factors. The prevalence of CKD is increasing by $8 \%$ annually worldwide, and patients with CKD have a predisposition to cardiovascular diseases (CVD) that manifest in different forms (coronary artery disease, stroke, etc.). The main cardiovascular risk factors are diabetes mellitus (DM), arterial hypertension, albuminuria, dyslipidaemia, and smoking (1). Herzog described a $44.4 \%$ incidence of typical chest pain in the group of dialysed patients in comparison with $68.3 \%$ incidence in the general population without CKD (2).

Non-invasive stress tests are the cornerstone in diagnosing coronary artery disease (CAD) in asymptomatic patients; sensitivity and specificity varies from test to test. ECG stress testing was commonly used because the available, meta-analysis of multiple studies showed a different sensitivity (23-100\%, mean $68 \%$ ) and specificity (17-100\%, mean $77 \%$ ) (4). Dialysed patients often cannot achieve an optimal heart rate, and thus pharmacologic stress testing should be performed. Dobutamine stress echocardiography (DSE) has a sensitivity from 75 to $95 \%$ and specificity from 76 to $94 \%$, with a CAD diagnosis accuracy of $90 \%$ (5). A negative DSE result identifies the low-risk population, with $97 \%$ absence of acute cardiovascular events or sudden cardiac death in $12 \pm 6$ 
Tab. 1. Basic characteristics of the patients in this study.

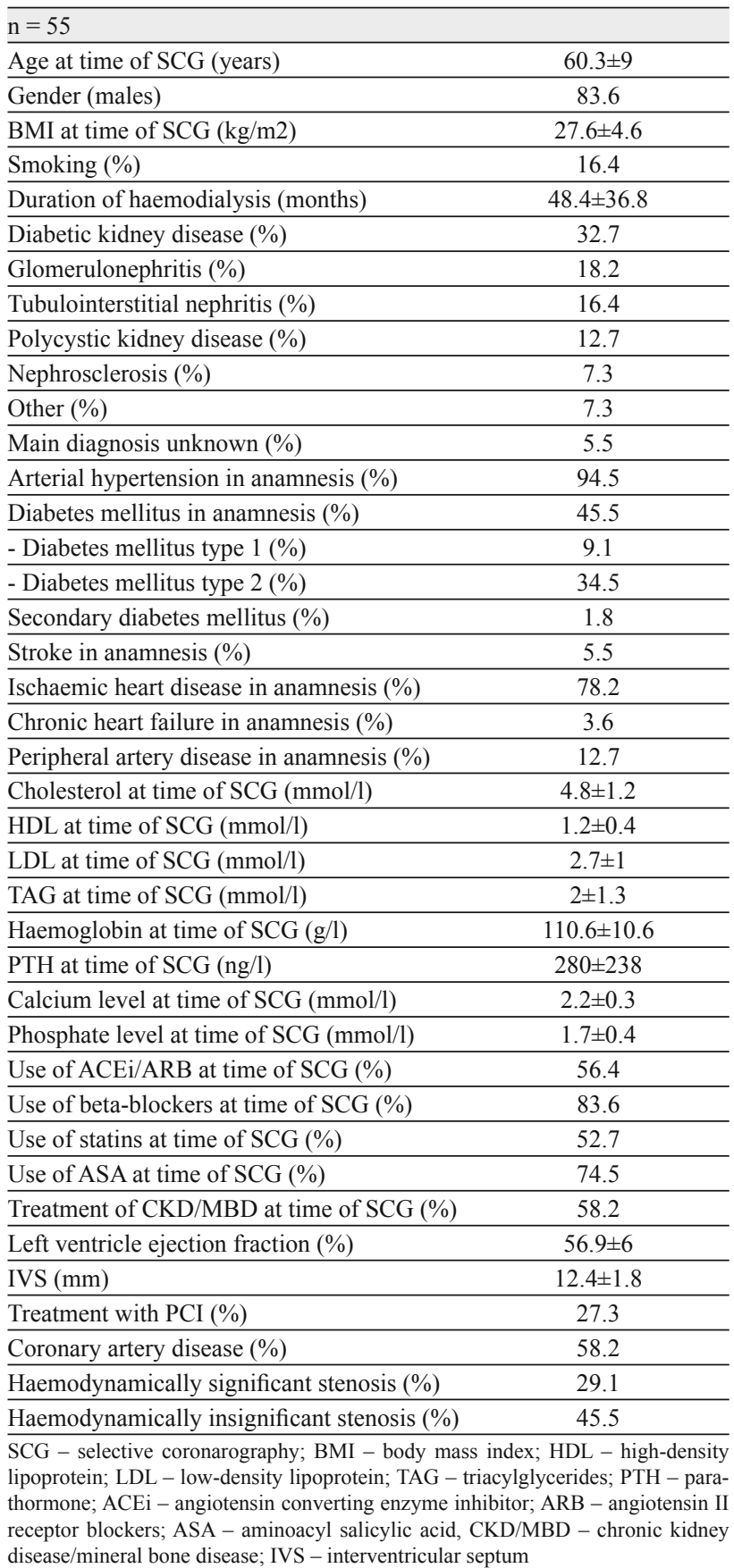

months (6). According to the ACC/AHA, in the general population, there is no need to perform stress testing in the patients with functional capacity $>10$ metabolic equivalents (METs). According to the European Society of Cardiology (ESC)/ European Society of Hypertension (ESH) guidelines, non-invasive stress testing should be performed in patients with poor functional capacity $(<4$ METs) with the presence of two or more risk factors (DM, anamnesis of cardiovascular disease, haemodialysis $>1$ year, age $>60$ years, arterial hypertension, left ventricular hypertrophy, dyslipidaemia)
(7). In the patients with end stage renal disease (ESRD) and a low functional capacity, only one risk factor is needed to perform noninvasive stress testing. If there are three or more risk factors, patients should undergo non-invasive stress testing regardless of the functional capacity (3). Coronary revascularisation should not be performed for the purpose of decreasing cardiovascular risk. The CARP study did not confirm differences in the survival between the patients treated pharmacologically and revascularized patients (8). Although the presence of haemodynamically (HD) significant stenosis of the coronary artery (lumen occlusion $>70 \%$ ) was a predictor of acute cardiovascular events in post-transplant period in the study of 126 kidney transplant candidates, $94 \%$ of the patients without CAD did not experience an acute cardiovascular event within 48 months. On the other hand, $54 \%$ of the patients with CAD did not experience an event in the same time frame (9).

Ramphul et al created a diagnostic algorithm for stratifying the patients into the risk groups: high risk patients are older than 60 years or younger than 60 years with at least one risk factor (DM, CAD, peripheral artery disease, heart failure), low risk patients are 40-60 years old and minimal risk patients are younger than 40 years. Based on the risk group, they chose to examine the modality. This stratification indicated that the patients with minimal risk have a $0.7 \%$ incidence of acute cardiovascular events annually, while the patients in very high-risk group have $8.9 \%$ (10). The recent study by Tabriziani stratified the patients into the risk groups based on the presence of risk factors, gender and age. A specific group with stricter criteria and diagnostic tests are the patients with DM with respect to duration and type of DM (11).

Kidney transplantation (KTx) is the best option for renal replacement therapy in the patients with ESRD. This modality improves survival, reduces associated complications, and improves the quality of life (12). When KTx is successful, patient's mortality is reduced. However, pre-existing CVD determine overall patient survival after KTx. For patients with low or medium cardiovascular risk, KTx is beneficial during the early post-transplant period, while the benefit of KTx in the patients with high cardiovascular risk is apparent from 6 to 12 months post-KTx (13). Therefore, it

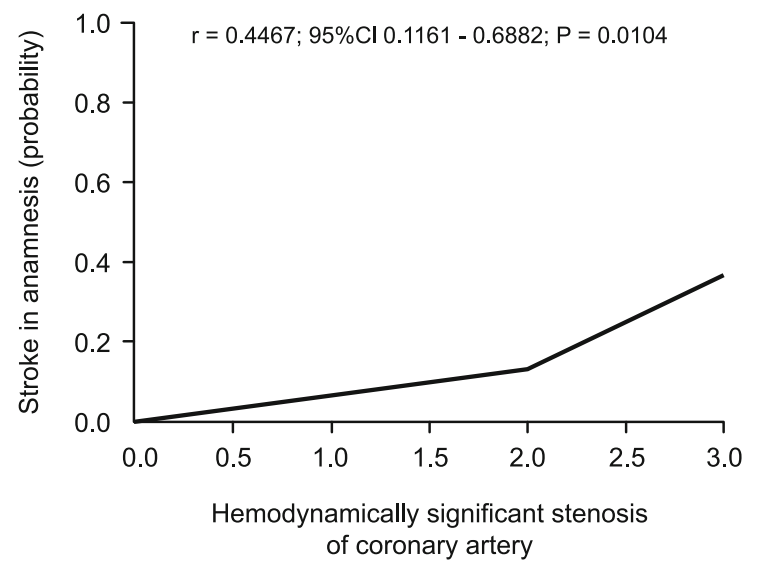

Fig. 1. Correlation between stroke and haemodynamically significant stenosis of the coronary arteries. 
Tab. 2. Comparison of the percutaneous coronary intervention (PCI) and control groups.

\begin{tabular}{|c|c|c|c|}
\hline & $\begin{array}{c}\text { Control group } \\
\mathrm{n}=40\end{array}$ & $\begin{array}{l}\text { PCI group } \\
\mathrm{n}=15\end{array}$ & $\mathrm{p}$ \\
\hline Age at time of SCG (years) & $60 \pm 9,5$ & $61.2 \pm 7.7$ & 0.6662 \\
\hline Gender (males) & 82.5 & 86.7 & 0.7102 \\
\hline BMI at time of SCG $(\mathrm{kg} / \mathrm{m} 2)$ & $27.7 \pm 4.6$ & $27.3 \pm 4.8$ & 0.7776 \\
\hline Smoking (\%) & 12.5 & 26.7 & 0.2091 \\
\hline Duration of haemodialysis (months) & $50.3 \pm 40$ & $43.3 \pm 27.5$ & 0.5359 \\
\hline Diabetic kidney disease (\%) & 25 & 53.3 & 0.0484 \\
\hline Glomerulonephritis (\%) & 17.5 & 20 & 0.8320 \\
\hline Tubulointerstitial nephritis (\%) & 15 & 20 & 0.6583 \\
\hline Polycystic kidney disease (\%) & 17.5 & 0 & 0.0857 \\
\hline Nephrosclerosis (\%) & 10 & 0 & 0.2076 \\
\hline Other $(\%)$ & 10 & 0 & 0.2076 \\
\hline Main diagnosis unknown (\%) & 5 & 6.7 & 0.8066 \\
\hline Arterial hypertension in anamnesis (\%) & 97.5 & 86.7 & 0.1193 \\
\hline Diabetes mellitus in anamnesis (\%) & 40 & 60 & 0.1887 \\
\hline Stroke in anamnesis $(\%)$ & 7.5 & 0 & 0.2798 \\
\hline Ischaemic heart disease in anamnesis (\%) & 70 & 100 & 0.0174 \\
\hline Chronic heart failure in anamnesis (\%) & 5 & 0 & 0.3820 \\
\hline Peripheral artery disease in anamnesis (\%) & 12.5 & 13.3 & 0.9347 \\
\hline Cholesterol at time of SCG $(\mathrm{mmol} / \mathrm{l})$ & $4.8 \pm 1.2$ & $4.8 \pm 1.1$ & 1.0000 \\
\hline HDL at time of SCG $(\mathrm{mmol} / \mathrm{l})$ & $1.3 \pm 0.4$ & $1.1 \pm 0.4$ & 0.1046 \\
\hline LDL at time of SCG $(\mathrm{mmol} / \mathrm{l})$ & $2.7 \pm 1$ & $2.8 \pm 1$ & 0.7425 \\
\hline TAG at time of SCG $(\mathrm{mmol} / \mathrm{l})$ & $2 \pm 1.4$ & $2.1 \pm 1.3$ & 0.8110 \\
\hline Haemoglobin at time of SCG (g/l) & $111 \pm 10.2$ & $109.7 \pm 12.1$ & 0.6908 \\
\hline PTH at time of SCG (ng/l) & $302 \pm 264$ & $224 \pm 151$ & 0.2867 \\
\hline Calcium level at time of SCG (mmol/l) & $2.2 \pm 0.2$ & $2.1 \pm 0.4$ & 0.2228 \\
\hline Phosphate level at time of SCG (mmol/l) & $1.8 \pm 0.5$ & $1.6 \pm 0.3$ & 0.1531 \\
\hline Use of ACEi/ARB at time of SCG (\%) & 60 & 46.7 & 0.3801 \\
\hline Use of beta-blockers at time of SCG (\%) & 85 & 80 & 0.6583 \\
\hline Use of statins at time of SCG (\%) & 50 & 60 & 0.5121 \\
\hline Use of ASA at time of SCG (\%) & 70 & 86.7 & 0.2095 \\
\hline Treatment of CKD/MBD at time of SCG (\%) & 60 & 53.3 & 0.6567 \\
\hline Left ventricle ejection fraction $(\%)$ & $57.1 \pm 6.3$ & $56.3 \pm 5.5$ & 0.6666 \\
\hline IVS (mm) & $12.5 \pm 2$ & $12.2 \pm 1.5$ & 0.6005 \\
\hline Coronary artery disease (\%) & 42.5 & 100 & 0.0001 \\
\hline
\end{tabular}

PCI - percutaneous coronary intervention; SCG - selective coronarography; BMI - body mass index; HDL high-density lipoprotein; LDL - low-density lipoprotein; TAG - triacylglycerides; PTH - parathormone; ACEi - angiotensin converting enzyme inhibitor; ARB - angiotensin II receptor AT1 blockers; ASA - 5-aminoacyl salicylic acid; IVS - interventricular septum

is crucial to assess the cardiovascular risk prior to kidney transplantation to anticipate or prevent acute cardiovascular events and maximize KTx benefits.

The primary aim for our study was to identify risk factors for CAD in the patients with ESRD prior to KTx. The secondary aims were to identify the effect of pharmacotherapy in developing CAD, identify low-risk patients that would allow a swift inclusion on the waiting list (WL) and recognize the risk factors that would contraindicate the patient listing on the WL or KTx.

\section{Materials and methods}

Our study was a retrospective analysis of the patients after KTx in the transplant centre in Martin, Slovakia. They underwent a diagnostic algorithm for CVD prior to KTx by undergoing a selective coronarography (SCG). At the time of coronarography, we obtained necessary information such as: age, gender, body mass index (BMI), smoking status, duration of haemodialysis, type of haemodialysis, cause of ESRD, other comorbidities (arterial hypertension, type of DM, stroke, ischaemic heart disease, chronic heart failure, peripheral artery disease), medication used (aminoacyl salicylic acid [ASA], statins, type of beta-blockers, drugs for $\mathrm{CKD} /$ mineral bone disease (MBD)). At the time of SCG, a blood sample was taken, and we monitored the levels of high-density lipoprotein cholesterol (HDL-C), lowdensity lipoprotein cholesterol (LDL-C), triacylglycerides (TAG), total cholesterol, haemoglobin and serum levels of total calcium, phosphate and parathormone (PTH). The patients underwent transthoracic echocardiogram (TTE) prior to coronarography. We monitored the following echocardiographic parameters: width of interventricular septum (IVS) and left ventricle ejection fraction with the biplane Simpson's method.

According to coronarography results, patients were divided into two groups: patients requiring revascularisation because of HD significant stenosis of the coronary artery, which was defined as arterial lumen occlusion $>75 \%$ (percutaneous intervention (PCI) group) and group of patients without HD significant stenosis of the coronary arteries (control group).

We used a certified statistical program, namely MedCalc version 13.1.2 (MedCalc Software VAT registration number BE 0809 344,640, Member of International Association of Statistical Computing, Ostend, Belgium). Comparisons of continuous variables between the groups were performed using parametric ( $t$-test) or non-parametric (Mann-Whitney) tests; associations between the categorical variables were analysed using the $\chi 2$ test and Fisher's exact test, as appropriate. Logistic regression was used for multivariate analysis for independent predictors of CAD. A p value $<$ 0.05 was considered to be statistically significant.

\section{Results}

A total of 55 patients (46 men and 9 women) were involved in this study. There were significantly more men than women ( $p$ $<0.0001)$. The mean patient age was $60.3 \pm 9$ years $(41-76$, median 61 years). A positive ECG stress test occurred in $10.9 \%$ of the patients, while $32.7 \%$ had a negative result and stress testing 


\section{$771-777$}

was not performed in $56.4 \%$ of the patients. HD significant stenosis of the coronary arteries was presents in 16 patients $(29.1 \%)$ : one vessel CAD (10 patients), two-vessel CAD (4 patients) and multiple-vessel CAD (2 patients). The basic characteristics of the patients in our study are shown in the Table 1.

There were no differences between the PCI and control groups with regards to age, gender, BMI or smoking status. In the PCI group, there was a statistically significant difference in the incidence of diabetic kidney disease $(p=0.0484)$, ischaemic heart disease in anamnesis $(p=0.0174)$ and CAD $(p=0.0001)$. There were no differences in biochemical parameters (lipid profile, calciumphosphate metabolism, level of haemoglobin), pharmacotherapy or echocardiographic parameters (left ventricular ejection fraction and IVS width) between the groups (Tab. 2).

We confirmed a positive correlation between the presence of stroke or transitory ischaemic attack (TIA) in personal anamnesis and incidence of HD significant stenosis of the coronary arteries (Fig. 1).

By using a logistic regression, we confirmed independent risk factors for HD significant stenosis of the coronary arteries (Tab. 3). Independent risk factors were: DM in the patient's history (odds ratio $(\mathrm{OR}) 2.3492 ; 95 \%$ confidence interval $(\mathrm{CI})=1.0105-5.4615$; $\mathrm{p}=0.0472)$, and levels of HDL cholesterol $<1.03 \mathrm{mmol} / \mathrm{l}(\mathrm{OR}$ 4.3276; 95\% CI = 1.1009-17.0123; $\mathrm{p}=0.0359)$, calcium $\leq 2.0$ $\mathrm{mmol} / 1$ (OR 2.4935; 95\% CI 1.0926-5.6905; $\mathrm{p}=0.0309)$ and phosphate $\geq 1.45 \mathrm{mmol} /$ (OR 0.2034; 95\% CI 0.0462-0.8946; $\mathrm{p}=0.0351)$

Tab. 3. Independent risk factors for haemodynamically significant coronary artery stenosis (logistic regression).

\begin{tabular}{|c|c|c|c|}
\hline PCI & Odds ratio & $95 \% \mathrm{CI}$ & $\mathrm{p}$ \\
\hline Age at time of $S C G \geq 60$ years & 1.1244 & $0.3337-3.7879$ & 0.8500 \\
\hline Gender (males) & 2.0264 & $0.3056-13.4391$ & 0.4644 \\
\hline BMI at time of $S C G \geq 30 \mathrm{~kg} / \mathrm{m} 2$ & 1.4966 & $0.3692-6.0661$ & 0.5723 \\
\hline Smoking & 2.5048 & $0.5049-12.4267$ & 0.2612 \\
\hline Duration of haemodialysis $\geq 60$ months & 0.5870 & $0.1202-2.8675$ & 0.5104 \\
\hline Diabetic kidney disease & 5.2276 & $0.4684-58.3489$ & 0.1790 \\
\hline Arterial hypertension in anamnesis & 0.1277 & $0.0093-1.7490$ & 0.1232 \\
\hline Diabetes mellitus in anamnesis & 2.3492 & $1.0105-5.4615$ & 0.0472 \\
\hline Stroke in anamnesis & 0.3456 & $0.01684-7.0948$ & 0.4908 \\
\hline Ischemic heart disease in anamnesis & 13.5965 & $0.7529-24.5386$ & 0.0771 \\
\hline Chronic heart failure in anamnesis & 0.4968 & $0.02253-10.9519$ & 0.6575 \\
\hline Peripheral artery disease in anamnesis & 1.1234 & $0.1349-9.3582$ & 0.9143 \\
\hline Cholesterol at time of SCG $\geq 5,17 \mathrm{mmol} / 1$ & 2.6385 & $0.6589-10.5666$ & 0.1705 \\
\hline HDL at time of $S C G \leq 1,03 \mathrm{mmol} / \mathrm{l}$ & 4.3276 & $1.1009-17.0123$ & 0.0359 \\
\hline LDL at time of $S C G \geq 3,3 \mathrm{mmol} / 1$ & 2.2197 & $0.4771-10.3270$ & 0.3094 \\
\hline TAG at time of SCG $\geq 1,7 \mathrm{mmol} / 1$ & 1.1556 & $0.3499-3.8163$ & 0.8125 \\
\hline Haemoglobin at time of $\mathrm{SCG}<110 \mathrm{~g} / 1$ & 2.1725 & $0.5679-8.3114$ & 0.2571 \\
\hline PTH at time of SCG $>300 \mathrm{ng} / 1$ & 0.4130 & $0.1016-1.6785$ & 0.2164 \\
\hline Ca at time of $\mathrm{SCG} \leq 2 \mathrm{mmol} / \mathrm{l}$ & 2.4935 & $1.0926-5.6905$ & 0.0309 \\
\hline$P$ at time of SCG $\geq 1,45 \mathrm{mmol} / \mathrm{l}$ & 0.2034 & $0.0462-0.8946$ & 0.0351 \\
\hline Ejection fraction of left ventricle $<50 \%$ & 0.8630 & $0.1645-4.5281$ & 0.8617 \\
\hline IVS $>12 \mathrm{~mm}$ & 1.4562 & $0.3653-5.8041$ & 0.5942 \\
\hline
\end{tabular}

PCI - percutaneous coronary intervention; SCG - selective coronarography; BMI - body mass index; HDL high-density lipoprotein; LDL - low density lipoprotein; TAG - triacylglycerides; PTH - parathormone; Ca calcium; P - phosphate; IVS - interventricular septum

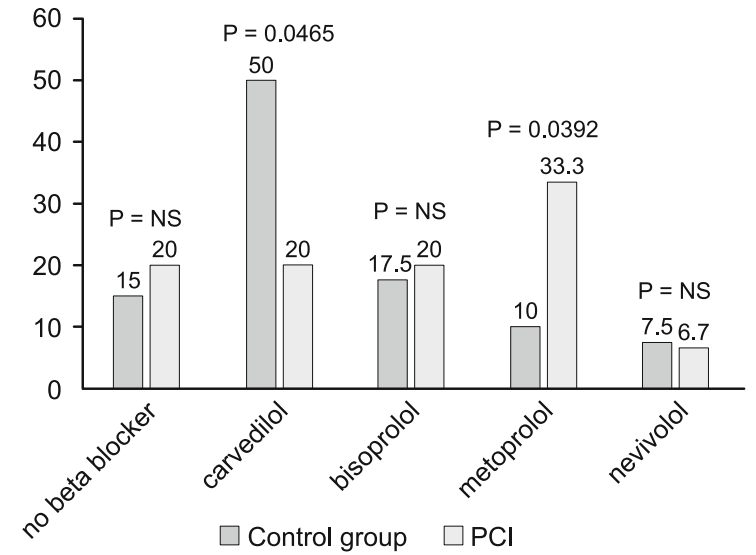

Fig. 2. Types of beta-blocker used in percutaneous coronary intervention (PCI) and control group patients.

There were significant differences between the groups with regards to the type of beta-blocker. Metoprolol use was significantly higher in the PCI group $(\mathrm{p}=0.0392)$, while carvedilol was used more often in the control group $(p=0.0465)$ (Fig. 2).

At the end of our study, we confirmed several risk factors that were potentially contraindicative for placement on the WL or for KTx. They included: smoking ( $p=0.0079)$, type 2 DM ( $p$ $=0.0087)$ and use of statins $(\mathrm{p}=0.0025)$. HD significant stenosis of the coronary arteries was not a statistically significant risk factor that contraindicated patients for KTx or not listing them on the WL (Tab. 4).

\section{Discussion}

CVD are the most common cause of mortality and complications after KTx. The risk of death in kidney transplant candidates (KTC) is 10-times higher, when they have CVD. Furthermore, this risk is 50-times higher than the mean nonfatal acute cardiovascular events, when compared to the general population. Notably, approximately one third of KTC have a significant stenosis of the coronary arteries $(14,15)$ Our study confirmed this fact because this dysfunction was present in $29.1 \%$ of the patients.

Our study confirmed independent risk factors for severe CAD. One of those risk factors was altered calcium-phosphate metabolism (serum level of calcium $\leq 2$ $\mathrm{mmol} / \mathrm{l}$ and serum level of phosphate $\geq 1.45$ $\mathrm{mmol} / \mathrm{l}$ ). Hyperphosphatemia is associated with increased risk of acute cardiovascular events in the patients with known CAD (16). In the observational retrospective study by Shin et al (17), the authors confirmed an association between hyperphosphatemia and 
Tab. 4. Comparison of patients on the kidney transplantation waiting list (Tx/WL-yes) and patients contraindicated transplantation $(\mathrm{Tx} / \mathrm{WL}-\mathrm{no})$.

\begin{tabular}{|c|c|c|c|}
\hline & $\begin{array}{c}\text { Tx/WL }- \text { yes } \\
n=43\end{array}$ & $\begin{array}{c}\mathrm{Tx} / \mathrm{WL}-\mathrm{no} \\
\mathrm{n}=12\end{array}$ & $\mathrm{p}$ \\
\hline Age at time of SCG (years) & $59.5 \pm 9.3$ & $63.3 \pm 7.2$ & 0.9168 \\
\hline Gender (males) & 83.7 & 83.3 & 0.9738 \\
\hline BMI at time of SCG $(\mathrm{kg} / \mathrm{m} 2)$ & $27.1 \pm 4.7$ & $29.4 \pm 4.1$ & 0.1301 \\
\hline Smoking (\%) & 2.3 & 25 & 0.0079 \\
\hline Duration of haemodialysis (months) & $49.3 \pm 39$ & $45.3 \pm 29.4$ & 0.7433 \\
\hline Diabetic kidney disease (\%) & 30.2 & 41.7 & 0.4569 \\
\hline Glomerulonephritis (\%) & 16.3 & 25 & 0.4937 \\
\hline Tubulointerstitial nephritis (\%) & 18.6 & 8.3 & 0.3980 \\
\hline Polycystic kidney disease (\%) & 11.6 & 16.7 & 0.6422 \\
\hline Nephrosclerosis (\%) & 7 & 8.3 & 0.8793 \\
\hline Other $(\%)$ & 9.3 & 0 & 0.2770 \\
\hline Main diagnosis unknown (\%) & 7 & 0 & 0.3503 \\
\hline Arterial hypertension in anamnesis (\%) & 93 & 100 & 0.3503 \\
\hline Diabetes mellitus in anamnesis (\%) & 39.5 & 66.7 & 0.0973 \\
\hline - Diabetes mellitus type $1(\%)$ & 11.6 & 0 & 0.2202 \\
\hline - Diabetes mellitus type $2(\%)$ & 25.6 & 66.7 & 0.0087 \\
\hline Secondary diabetes mellitus (\%) & 2.3 & 0 & 0.5994 \\
\hline Stroke in anamnesis (\%) & 2.3 & 16.7 & 0.0540 \\
\hline Ischaemic heart disease in anamnesis (\%) & 74.4 & 91.7 & 0.2037 \\
\hline Chronic heart failure in anamnesis (\%) & 2.3 & 8.3 & 0.3289 \\
\hline Peripheral artery disease in anamnesis (\%) & 9.3 & 25 & 0.1528 \\
\hline Cholesterol at time of SCG (mmol/l) & $4.9 \pm 1.1$ & $4.7 \pm 1.4$ & 0.6023 \\
\hline HDL at time of SCG $(\mathrm{mmol} / \mathrm{l})$ & $1.3 \pm 0.4$ & $1.1 \pm 0.3$ & 0.1142 \\
\hline LDL at time of SCG (mmol/l) & $2.8 \pm 1$ & $2.6 \pm 1.1$ & 0.5428 \\
\hline TAG at time of SCG $(\mathrm{mmol} / \mathrm{l})$ & $1.9 \pm 1.4$ & $2.3 \pm 1$ & 0.3600 \\
\hline Haemoglobin at time of SCG (g/l) & $111 \pm 11.5$ & $108.8 \pm 6,6$ & 0.5304 \\
\hline PTH at time of SCG (ng/l) & $308 \pm 259$ & $184 \pm 107$ & 0.1130 \\
\hline Calcium level at time of SCG $(\mathrm{mmol} / \mathrm{l})$ & $2.2 \pm 0.2$ & $2.1 \pm 0.5$ & 0.2942 \\
\hline Phosphate level at time of SCG (mmol/l) & $1.7 \pm 0.5$ & $1.7 \pm 0.4$ & 1.0000 \\
\hline Use of ACEi/ARB at time of SCG (\%) & 60.5 & 41.7 & 0.2499 \\
\hline Use of beta-blockers at time of SCG (\%) & 79.1 & 100 & 0.0862 \\
\hline Use of statins at time of SCG (\%) & 41.9 & 91.7 & 0.0025 \\
\hline Use of ASA at time of SCG $(\%)$ & 76.7 & 66.7 & 0.4861 \\
\hline Treatment of CKD/MBD at time of SCG (\%) & 55.8 & 66.7 & 0.5024 \\
\hline Left ventricle ejection fraction (\%) & $56.8 \pm 5.6$ & $57.2 \pm 7.6$ & 0.8408 \\
\hline IVS (mm) & $12.4 \pm 1.9$ & $12.7 \pm 1.8$ & 0.6270 \\
\hline Treatment with PCI (\%) & 55.8 & 66.7 & 0.5024 \\
\hline Coronary artery disease $(\%)$ & 27.9 & 25 & 0.8433 \\
\hline Haemodynamically significant stenosis (\%) & 30.2 & 33.3 & 0.8386 \\
\hline Haemodynamically insignificant stenosis (\%) & 44.2 & 50 & 0.7237 \\
\hline
\end{tabular}

PCI - percutaneous coronary intervention; SCG - selective coronarography; BMI - body mass index; HDL high-density lipoprotein; LDL - low density lipoprotein; TAG - triacylglycerides; PTH - parathormone; Ca calcium; P - phosphate; ACEi - angiotensin converting enzyme inhibitor; ARB - angiotensin II receptor AT1 blockers; ASA - 5-aminoacyl salicylic acid; IVS - interventricular septum

HDL-C particles enzymatically remove oxidised LDL particles (which increase atherogenicity). CAD development increases, when LDL-C levels are increased concomitant with reduced HDL-C (18). Our study revealed that HDL-C $\leq 1.03 \mathrm{mmol} / \mathrm{l}$ was an independent risk factor of HD significant stenosis of the coronary arteries $(\mathrm{p}=$ 0.0359). Patients with low HDL-C have increased risk of CAD, in stent re-stenosis after PCI and mortality of cardiovascular causes, particularly if the patients are males and have DM (19). The retrospective study of Kashayar and Mohagehi demonstrated that even with effective LDL-C decrease to therapeutic levels, in the long term, elevating HDL-C should be a priority to prevent cardiovascular mortality (20).

Our study revealed that DM was an independent risk factor for severe CAD ( $p$ $=0.0359$ ). The presence of DM worsened cardiovascular prognosis 3-fold compared with the non-diabetic population (21). According to the NHANES study, the prevalence of diabetic kidney disease (DKD) is increasing, and it is one of the most common causes of ESRD. Our data confirmed this finding because DKD was the most common cause of ESRD (32.7 \%) with a significantly increased incidence in the PCI group (53\% versus $25 \%$ in the control group, $p=0.0484)$. This difference can be explained by the DM duration. In the study of 175 patients, the authors confirmed macrovascular complications, including coronary artery calcification and number and degree of affected coronary arteries were time dependent with its stabilization over 5 to 10 years (22).

In our analysis, there was a positive link between stroke (or TIA) in anamnesis and HD significant stenosis of the coronary arteries $(\mathrm{p}=0.0104)$. Risk factors for both diseases (stroke and CAD) were similar, but not the same. Patients with anamnesis of stroke and severe intracranial atherosclerosis also had an increased incidence of dyslipidaemia, metabolic syndrome, and

increased coronary calcification in patients with CKD as well as in patients with a preserved renal function. This study confirmed independent risk factors (serum levels of calcium, phosphate, and calcium-phosphate products) for coronary atherosclerosis, particularly in patients with DM, where advanced glycated end products (AGEP) enhanced mineralisation and CAD incidence on the background of mineral imbalance (17). type 2 DM (23). Hypothetically, if the severity of coronary artery atherosclerosis is analogous with cerebral artery atherosclerosis, in our study type $2 \mathrm{DM}(\mathrm{p}=0.0472)$ and dyslipidaemia (low HDLcholesterol, $\mathrm{p}=0.0359$ ) would contribute to the development of severe CAD in the PCI group. In the AMISTAD study, authors observed an association between $\mathrm{a} \geq 50 \%$ occurrence of coronary artery stenosis (verified by coronarography) in $26 \%$ of the patients 
with asymptomatic CAD and stroke of different aetiologies; $62 \%$ of the patients had borderline insignificant atherosclerotic changes of coronary arteries (24). In the AMISTAD substudy, the authors revealed a 2-fold higher risk of acute cardiovascular events in the patients, who had suffered non-fatal ischaemic stroke and had asymptomatic CAD diagnosed by coronarography (25).

Our retrospective analysis found a difference between betablockers used in the PCI and the control groups. In the PCI group (with more severe CAD), the cardio selective beta-blocker (metoprolol) was used more often (33.3 versus $10 \%$ for the control, $p=$ 0.0392). Metoprolol increases insulin resistance and causes hyperinsulinemia. This dysregulation leads to functional and structural changes in coronary arteries (26). In the control group, carvedilol was used more often $(50 \%$ versus $20 \%$ for the PCI group, $\mathrm{p}=$ $0.0465)$. In the COMET study, type $2 \mathrm{DM}$ development was reduced by $22 \%$ in the patients using carvedilol compared to metoprolol in 5 -year follow-up (27). In the randomised, double-blind study, the authors compared the effect of metoprolol and carvedilol in the patients with type $2 \mathrm{DM}$ and arterial hypertension and observed a significant reduction in the risk of albuminuria progression and better compensated DM with lower levels of glycated haemoglobin in the patients using carvedilol (28). These data are consistent with the finding in our study: low levels of HDL-C and type 2 DM are risk factors for HD significant stenosis of the coronary arteries.

In our study, smoking was identified as a risk factor that might potentially contraindicate patients for placement on the KTx WL $(p=0.0079)$. Smoking is not an absolute contraindication for KTx. It is a traditional risk factor for CVD: smokers have a higher risk of generalised atherosclerosis that affects carotid or lower limb circulation. An increased risk of incidence of comorbidities delays listing KTC on the KTx WL due to diagnostic and therapeutic interventions. Myriad studies confirmed the negative effect of smoking on graft survival and survival of the patients (29).

Type 2 DM is the most common type of DM. Its associated micro- and macrovascular complications lead to functional and structural changes of vessels. Its presence prolongs the time for placing a patient on the KTx WL because it is necessary to perform diagnostic methods to unmask asymptomatic comorbidities. In our study, a significant number of patients were contraindicated or not listed for KTx due to the presence of type $2 \mathrm{DM}(\mathrm{p}=0.0087)$.

Type $2 \mathrm{DM}$ - along with dyslipidaemia - is part of metabolic syndrome. It is an independent risk factor for developing acute cardiovascular events, which was confirmed in the study of 337 patients after KTx, with $32 \%$ prevalence of metabolic syndrome $(p=0.002)(30)$. Statins were more often used in the risk group for $\operatorname{KTx}(\mathrm{p}=0.0025)$, meaning that patients in this group were more often treated for dyslipidaemia. Although there was no difference in LDL-C levels (for which statins are used to decrease), patients listed on the KTx WL, who already underwent KTx did not have at least two risk factors of metabolic syndrome.

\section{Conclusion}

Patients with ESRD have a very high risk for developing cardiovascular events. KTx is the most beneficial type of renal re- placement therapy, and it improves patient survival. To maximally benefit from KTx, clinicians must identify potential asymptomatic comorbidities and risk factors. CVD represent the most common cause of complications and mortality of the patients after KTx, which can compromise the maximal benefit from KTx. Diagnosis of CAD in ESRD is complicated due to incoherent signs and symptoms in anamnesis, physical findings, echocardiographic abnormalities, and low prediction value of stress testing. Potential revascularization in the patients with suspected CAD is sometimes a long and complicated process. A prolonged time spent in dialysis worsens the prognosis of patient and graft function after KTx. Thus, the goal of transplantation centres is to shorten the time interval between beginning of dialysis and listing patients on the WL. For this purpose, it is necessary to identify high-risk groups of the patients by analysing the presence of risk factors with incidence of comorbidities directly with SCG. On the other hand, identifying low risk patients with a low probability of CAD can quicken their placement on the WL without having to perform unnecessary stress testing.

\section{References}

1. Charrois TL, Zolezzi M, Koshman SL et al. A systematic review of the evidence for pharmacist care of patients with dyslipidemia. Pharmacotherapy 2012; 32: 222-233.

2. Herzog CA, Littrell K, Arko C et al. Clinical characteristics of dialysis patients with acute myocardial infarction in the United States: a collaborative project of the United States Renal Data System and the National Registry of Myocardial Infarction. Circulation 2007; 116: 1465-1472.

3. Lentine KL, Costa SP, Weir MR et al. Cardiac disease evaluation and management among kidney and liver transplantation candidates: a scientific statement fromthe American Heart Association and the American College of Cardiology Foundation: endorsed by the American Society of Transplant Surgeons, American Society of Transplantation, and National Kidney Foundation. Circulation 2012; 126: 617-663.

4. Gibbons RJ, Abrams J, Chatterjee K et al. ACC/AHA 2002 guideline update forthe management of patients with chronic stable angina: summary article: a report of the American College of Cardiology/American Heart Association Task Force on Practice Guidelines (Committee on the Management of Patients With Chronic Stable Angina). Circulation 2003; 107: $149-158$.

5. Reis G, Marcovitz PA, Leichtman AB et al. Usefulness of dobutamine stress echocardiography in detecting coronary artery disease in end-stage renal disease. Am J Cardiol 1995; 75: 707-710.

6. Sharma R, Pellerin D, Gaze DC et al. Dobutamine stress echocardiography and cardiac troponin $\mathrm{T}$ for the detection of significant coronary artery disease and predicting outcome in renal transplant candidates. Eur J Echocardiogr 2005; 6: 327-335.

7. Kristensen SD, Knuuti J, Saraste A et al. 2014 ESC/ESA Guidelines on non-cardiac surgery: cardiovascular assessment and management: the Joint Task Force on noncardiac surgery: cardiovascular assessment and management of the European Society of Cardiology (ESC) and the European Society of Anaesthesiology (ESA). Eur Heart J 2014; 35: 2383-2431.

8. McFalls EO, Ward HB, Moritz TE et al. Coronary-artery revascularization before elective major vascular surgery. N Engl J Med 2004; 351: 2795-2804. 
9. De Lima JJ, Sabbaga E, Vieira MLC et al. Coronary angiography is the best predictor of events in renal transplant candidates compared with noninvasive testing. Hypertension 2003; 42: 263-268.

10. Ramphul R, Fernandez M, Firoozi S et al. Assessing cardiovascular risk in chronic kidney disease patients prior to kidney transplantation: clinical usefulness of a standardised cardiovascular assessment protocol. BMC Nephrol. 2018; 19 (1): 2.

11. Tabriziani H, Baron P, Abudayyeh I et al. Cardiac risk assessment for end-stage renal disease patients on the renal transplant waiting list. Clin Kid J 2019; 12 (4): 576-585.

12. Loubeau PR, Loubeau JM, Jantzen R. The economics of kidney transplantation versus hemodialysis. Prog Transplant 2001; 11: 291-297.

13. Wolfe R, Ashby V, Milford $\mathbf{E}$ et al. Comparison of mortality in all patients on dialysis, patients on dialysis awaiting transplantation, and recipients of a first deceased-donor transplant. N Engl J Med1999; 341: $1725-1730$.

14. Liefeldt L, Budde K. Risk factors for cardiovascular disease in renal transplant recipients and strategies to minimize risk. Transpl Int 2010; 23: 1191-1204.

15. De Lima JJ, Gowdak LH, de Paula FJ et al. Influence of coronary artery disease assessment and treatment in the incidence of cardiac events in renal transplant recipients. Clin Transplant 2010; 24: 474-480.

16. Kestenbaum B, Sampson JN, Rudser KD et al. Serum phosphate levels and mortality risk among people with chronic kidney disease. J Am SocNephrol 2005; 16: 520-528.

17. Yamagishi S, Fujimori H, Yonekura $\mathbf{H}$ et al. Advanced glycation end products accelerate calcification in microvascular pericytes. Biochem Biophys Res Commun1999; 258: 353-357.

18. Tarchalski J, Guzik P, Wysocki $\mathbf{H}$ et al. Correlation between the extent of coronary atherosclerosis and lipid profile. Mol Cell Biochem 2003; 246: 25-30.

19. Ashen MD, Blumenthal RS. Low HDL cholesterol level. N Eng J Med 2005; 353: 1252-1260.
20. Khashayar P, Mohagheghi A. The correlation between dyslipidemia and coronary artery disease based on angiographic findings in an Iranian population. Acta Med Indones-Indones J Intern Med 2010; 42 (2): 82-85.

21. Srikanth S, Deedwania P. Management of coronary artery disease in patients with type 2 diabetes mellitus. CurrCardiol Rep 2007; 9: 264-271.

22. Srinsivan MP, Kamath PK, Bhat NM et al. Severity of coronary artery disease in type 2 diabetes mellitus: Does the timing matter? Indian Heart J 2016; 168: 158-163.

23. Arenillas JF. Intracranial atherosclerosis: current concepts. Stroke 2011; 42 (Suppl): S20-S23.

24. Amarenco P, Lavallée PC, Labereuche L et al. Prevalence of coronary atherosclerosis in patients with cerebral infarction. Stroke 2011; 42: $22-29$.

25. Amarenco $\mathbf{P}$, Levallée PC, Labereuche $\mathbf{L}$ et al. Coronary artery disease and risk of major vascular events after cerebral infarction. Stroke 2013; 44: 1505-1511.

26. Mather KJ, Steinberg HO, Baron AD. Insulin resistance in the vasculature. J Clin Invest 2013; 123: 2013-2014.

27. Poole-Wilson PA, Swedberg K, Cleland JG et al. Comparison of carvedilol and metoprolol on clinical outcomes in patients with chronic heart failure in the Carvedilol or Metoprolol European Trial (COMET): randomised controlled trial. Lancet 2003; 362: 7e13.

28. Bakris GL, Fonseca V, Katholi RE et al. Metabolic effects of carvedilol vs metoprolol in patients with type 2 diabetes mellitus and hypertension: a randomized controlled trial. JAMA 2004; 292: 2227e2236.

29. Aref A, Sharma A, Halawa A. Smoking in renal transplantation: facts beyond myth. World J Transplant 2017; 7 (2): 129-133.

30. Courivaud C, Kazory A, Simula-Faivre D et al. Metabolic syndrome and atherosclerotic events in renal transplant recipients. Transplantation 2007; 83: 1577-1581.

Received April 9, 2021. Accepted April 14, 2021. 